\title{
Solar Cycle in Photosphere and Corona
}

\author{
Elena E. Benevolenskaya ${ }^{1,2}$ \\ ${ }^{1}$ Stanford University, Stanford, CA 94305, USA USA email: elena@quake.stanford.edu \\ ${ }^{2}$ Pulkovo Astronomical Observatory, St. Petersburg, Russia
}

\begin{abstract}
The solar cycle in photosphere and corona reflects the internal dynamics on the Sun. According to the modern understanding the observed behavior of solar activity is related to the dynamo process in convection zone and photosphere and it is closely connected with the evolution of the coronal structures. The observational data from ground and space allow us to investigate the solar cycle as a complex phenomenon and to clarify the most important features such as polar magnetic field reversals, non-uniform distributions of the solar activity complexes and fine structure of the solar cycle.
\end{abstract}

\section{Introduction}

The solar magnetic cycle consists of two 11-years cycles of sunspot activity and affects all levels of the Sun (the convection zone, photosphere, chromosphere and corona). It is observed in the photosphere as a change of the order of magnetic polarity in active regions and complexes of solar activity from one 11-years cycle to another (Hale's law).

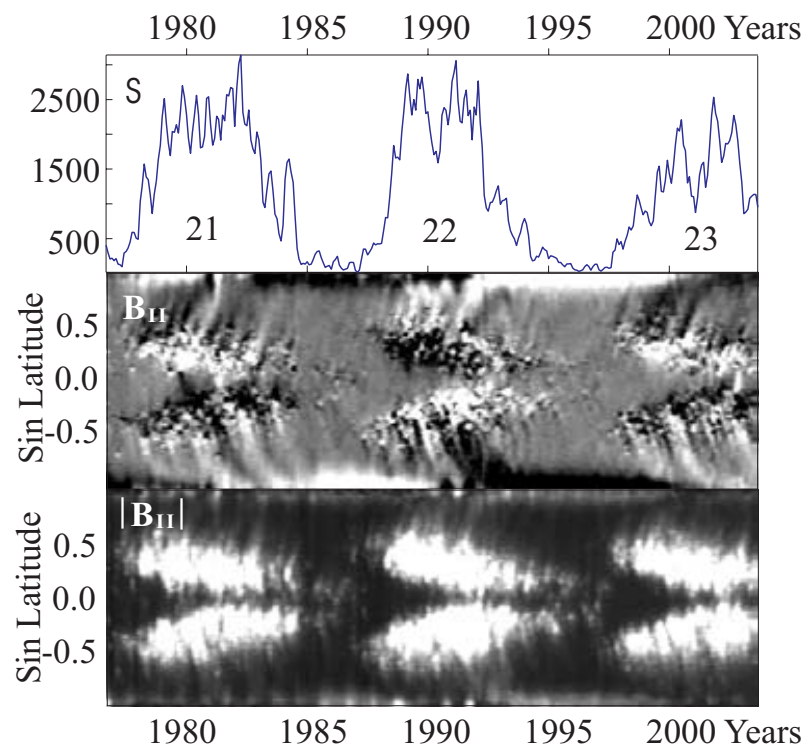

Figure 1. a) Sunspot area (3-months averaged) (S), in solar cycle 21, 22, 23 ; Axisymmetrical distributions of (b) the line-of-sight component of magnetic field strength $B_{\|}$in the range [-5G 5G] and (c)unsigned magnetic flux $\left|B_{\|}\right|$of $[020 \mathrm{G}]$.

It reveals a non-uniform latitudinal distribution of sunspots or so-called 'butterfly' diagram (Spörer's law). For example, Figure 1 illustrates the sunspot and magnetic activity during the last three cycles. The sunspot area $(\mathbf{S})$ reflects the level of sunspot activity and displays the 11-years periodicity (Figure 1a). The net magnetic flux or line-of-sight component of the magnetic strength $\left(B_{\|}\right)$displays the 22-years periodicity (Figure $1 \mathrm{~b}$ ). 
The positive polarity is marked by white color and negative polarity is marked by black color. The unsigned magnetic flux $\left(\left|B_{||}\right|\right)$demonstrates 'butterfly'-type distributions.

It is assumed that the cyclic behavior of the solar magnetic field is associated with dynamo processes in the solar interior. During a solar magnetic cycle, which typically lasts 22 years, the magnetic topology changes from largely poloidal field in the activity minima to predominantly toroidal field in the maxima. Magnetic polarity of the fields is reversed every 11 years. The toroidal field forms bipolar active regions and sunspots in low-latitude zones which migrate from approximately $30^{\circ}$ latitude towards the equator during the 11-year sunspot cycles (or magnetic half-cycles) forming the famous 'butterfly' diagram.

The topological model of the solar cycle suggested by Babcock (1961) introduced two main elements of the cycle: generation of the toroidal field by the solar differential rotation from the poloidal field and regeneration of the poloidal field due to poleward migration of the following magnetic polarities of active regions and reconnection in the corona across the equator. This results in reversal of the polar field polarities around the sunspot maxima. Following Babcock's ideas Leighton (1969) and Wang et al. (1991) developed quantitative models of the poleward migration due to diffusion and meridional circulation, which can closely reproduce the dynamics of the surface fields by adjusting the speed of meridional circulation and the diffusion coefficient. Theoretical investigations led to a suggestion that the poloidal field is likely to be generated by cyclonic convection ('alpha-effect') in the convection zone (Parker, 1955). This model can also explain the equatorward migration of the magnetic zones ('dynamo waves').

\section{Second Maximum of the Solar activity}

Such a very interesting feature as a double peak of the solar activity is still a puzzle, also, since finding of the pronounced second peak in solar cycle in green corona $(5303 \AA)$ in cycle 19. The first had coincided with maximum of sunspot number and, corona had flashed on the whole limb. The second was two year latter and corona became brighter at latitudes approximately equal to 15 (Gnevyshev, 1967).

It should be mentioned that Waldmeier (1957) found evidence of a double periodicity in the coronal emission, with Maxima occurring both at sunspot maximum and again near sunspot Minimum. "In addition to the strong maxima at sunspot latitudes, however there are small maxima, frequently almost completely lost in the wings of the strong maxima in intensity plots, but of a great deal of interest."

The double peak structure was pronounced in the distributions of large sunspot groups with areas are more than $500 \mathrm{mph}$ and distribution of the solar flares. Usually, temporal distance between these maxima equals approximately two years. On the base of the investigation of sunspot activity Antalova and Gnevyshev (1985) had suggested that solar cycle may be consisted of several impulses of solar activity with periods about $0.5-2.0$ years.

The evolution of the EUV fluxes (in Fe IX,X (171 $\AA$ ), Fe XII (195 ^) and Fe XV $(284 \AA)$ lines) averaged over latitude are plotted in Figure $2(d, e, f)$. For comparison, the indexes of solar activity: the relative sunspot number (Wolf number), the sunspot area and the $10.7 \mathrm{~cm}$ radio emission from the full disk are placed in Figure $2(a, b, c)$. The EUV emission in line Fe XV $(284 \AA)$ shows the pronounced double peak pattern to compare with both another lines. The first and the second maxima of the current cycle 23 are marked by 'I' and 'II'. The second maximum has occurred 1.5 years after the first peak. It is also clearly seen that the first maximum is more pronounced in the sunspot number and the second is greater in the sunspot area. 


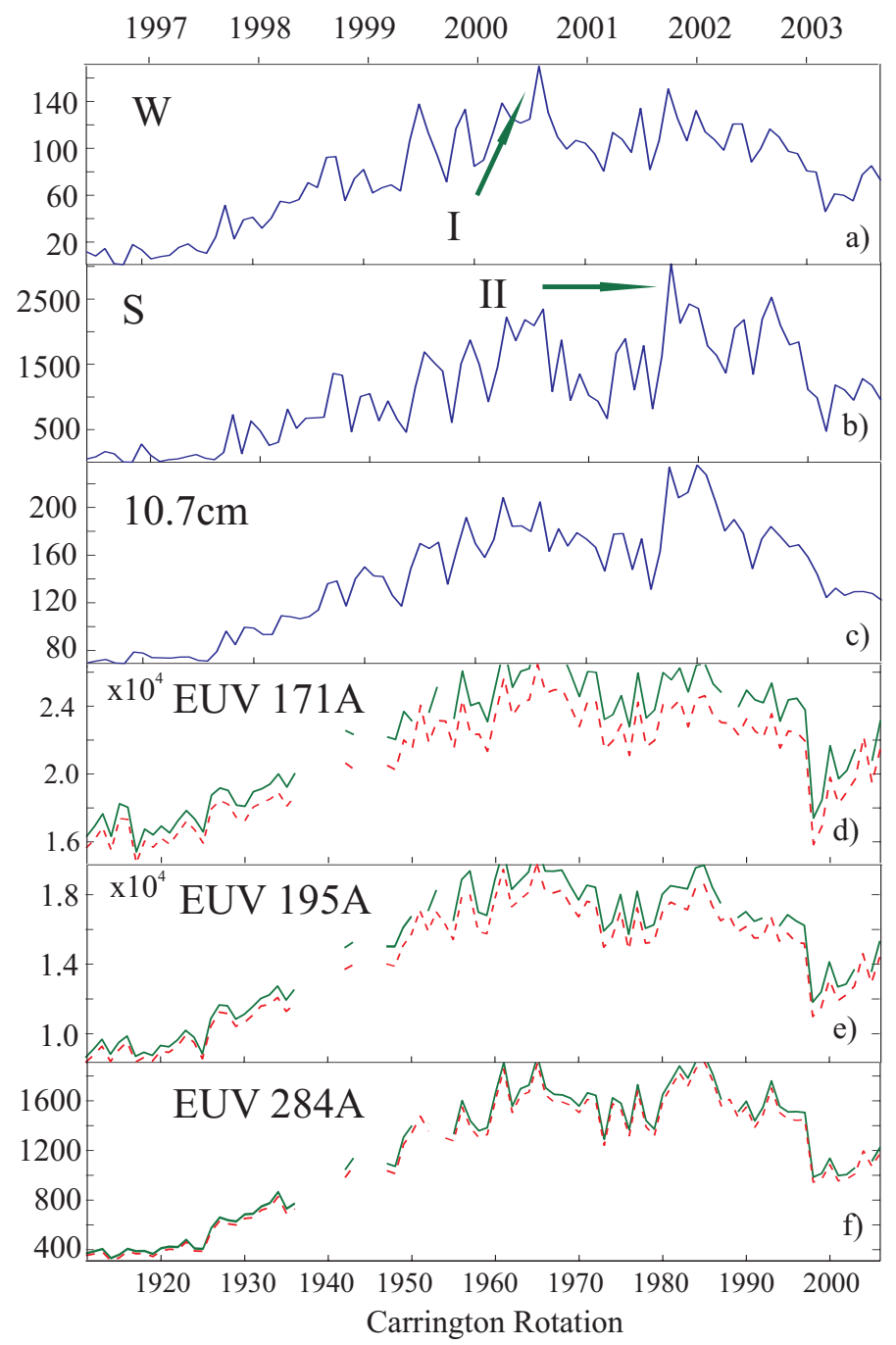

Figure 2. The upper three panels show the standard characteristics of solar activity: a) the Wolf's sunspot number; b) the total sunspot area; and c) $10.7 \mathrm{~cm}$ radio emission from the full disk. In panels a) and b), the first and second solar maxima are marked as 'I' and 'II'. The lower panels show the corresponding variations of the averaged over latitudes coronal EUV emissions: in Fe IX,X (171 ̊) (panel d), Fe XII (195 A)(panel e) and Fe XV (284 ̊) (panel f) lines. Dash line defines the coronal emission averaged over $-70^{\circ}$ to $70^{\circ}$, and solid line does for the the coronal emission averaged over $-75^{\circ}$ to $75^{\circ}$

\section{Polar magnetic field reversals and Double magnetic cycle}

It was noted that polar magnetic field reversals could be the singe or the three-fold. During the three-fold polar magnetic field reversal the temporal separation of the zones of alternated polarity of the magnetic field, determined from $\mathrm{H}$-alpha synoptic charts is closely to 1.5-2.5 years (Makarov and Sivaraman, 1989). In order to explain this phenomenon in frame of the dynamo theory it was suggested that the solar magnetic cycle consists of two main periodicities: a low-frequency component (Hale's 22 year cycle) and a high-frequency component (quasi-biennial cycle) (Benevolenskaya, 1991). The threefold polar magnetic field reversals mostly occur during the even cycles in one of the 

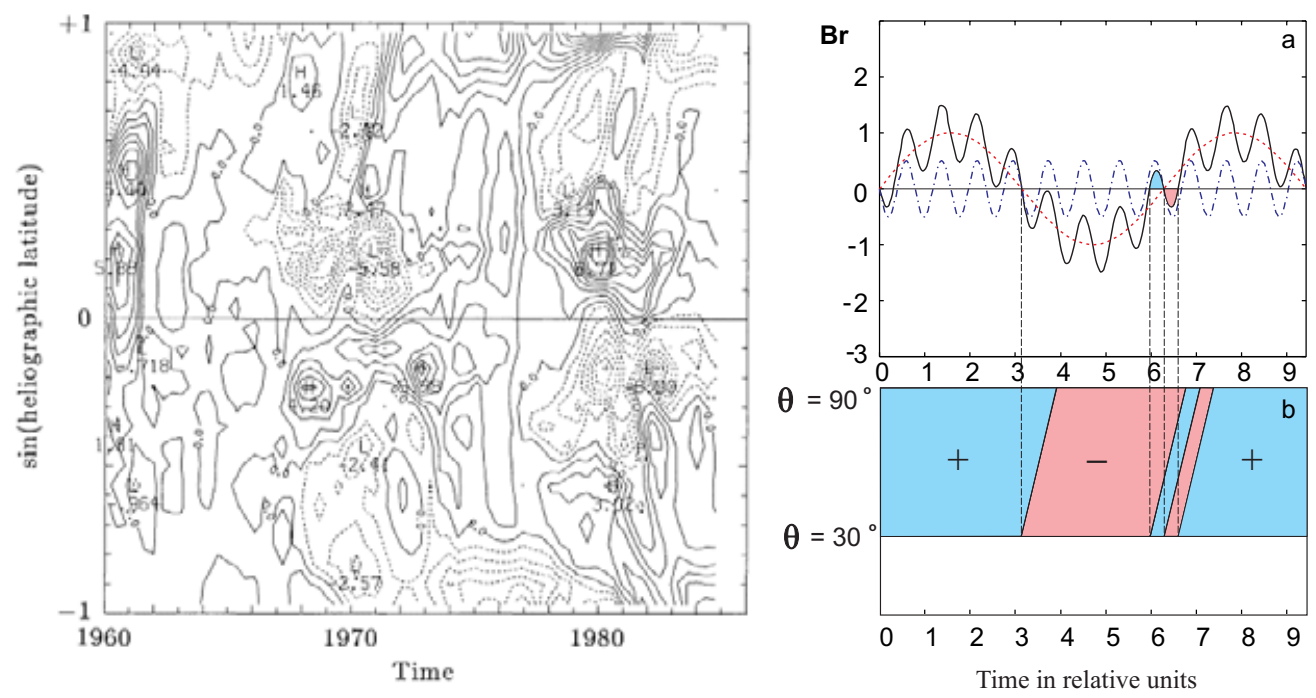

Figure 3. Left Panel: Axisymmetrical distribution of the magnetic field (Figure 2 from (Stenflo, 1988). Right panel: Scheme of the three-fold polar magnetic field reversal

hemispheres when the north polar magnetic field changes polarity from - to + and the south magnetic field from + to - . This defines the phase between two components (high-frequency and low-frequency) to be $\varphi=\pi$.

The simulated magnetic field, $B_{r}$, at the some fixed latitude, $\theta_{c}$, is illustrated in Figure 3(right panel) in case: $\varphi=\pi, A=2.2$ and amplitude's ratio is 8 . From this figure we note that the low-frequency change of polarity from - to + in northern hemisphere, the high-frequency wave can apper with zones of alternating polarities. The evolution of the axi-symmetrical or zonal magnetic flux is shown in Figure 3(left panel), where negative polarity is marked by dash line and positive polarity is done by solid line.

The high-frequency component is clearly seen in the latitude-time diagram of the line-of-sight component of the magnetic flux as a latitudinally extended structures of alternating polarity from low to higher latitudes in Figure 1(b) or 'surges' according to Sheeley and Wang (1989).

The existence of three-fold polar magnetic field reversals and zones of alternating polarity in the line-of-sight component of the magnetic field, the two pronounced peaks pattern in time series of long-lived complexes of solar activity may be considered as an evidence of the existence of the high-frequency component of the magnetic field together with the 22-years solar magnetic cycle. All these facts led us to hypothesis of 'Double magnetic cycle' (Benevolenskaya, 1991) and to the idea about the generation both cycle in convection zones (Benevolenskaya, 1998). And, thus, the conception of the 'Double magnetic cycle' explains 'Impulses of solar activity' visible in sunspot's distributions and in coronal brightness.

\section{Investigation of the solar cycle in the corona}

There is a long history of coronal investigation (Secchi, 1877; d'Azambuja, 1945; Waldmeier, 1957; Leroy and Trellis, 1974; Rǔsin, et al. 1990; Wilson, 1988). These studies found equatorward and poleward migrating bright structures in coronal emissions, which sometimes are called 'coronal activity waves', and thought to be related to dynamo waves which describe latitudinal migration of magnetic zones.

These investigations inspired suggestions that the polar waves of activity might be associated with another dynamo wave in the convection zone and that, in fact, the 


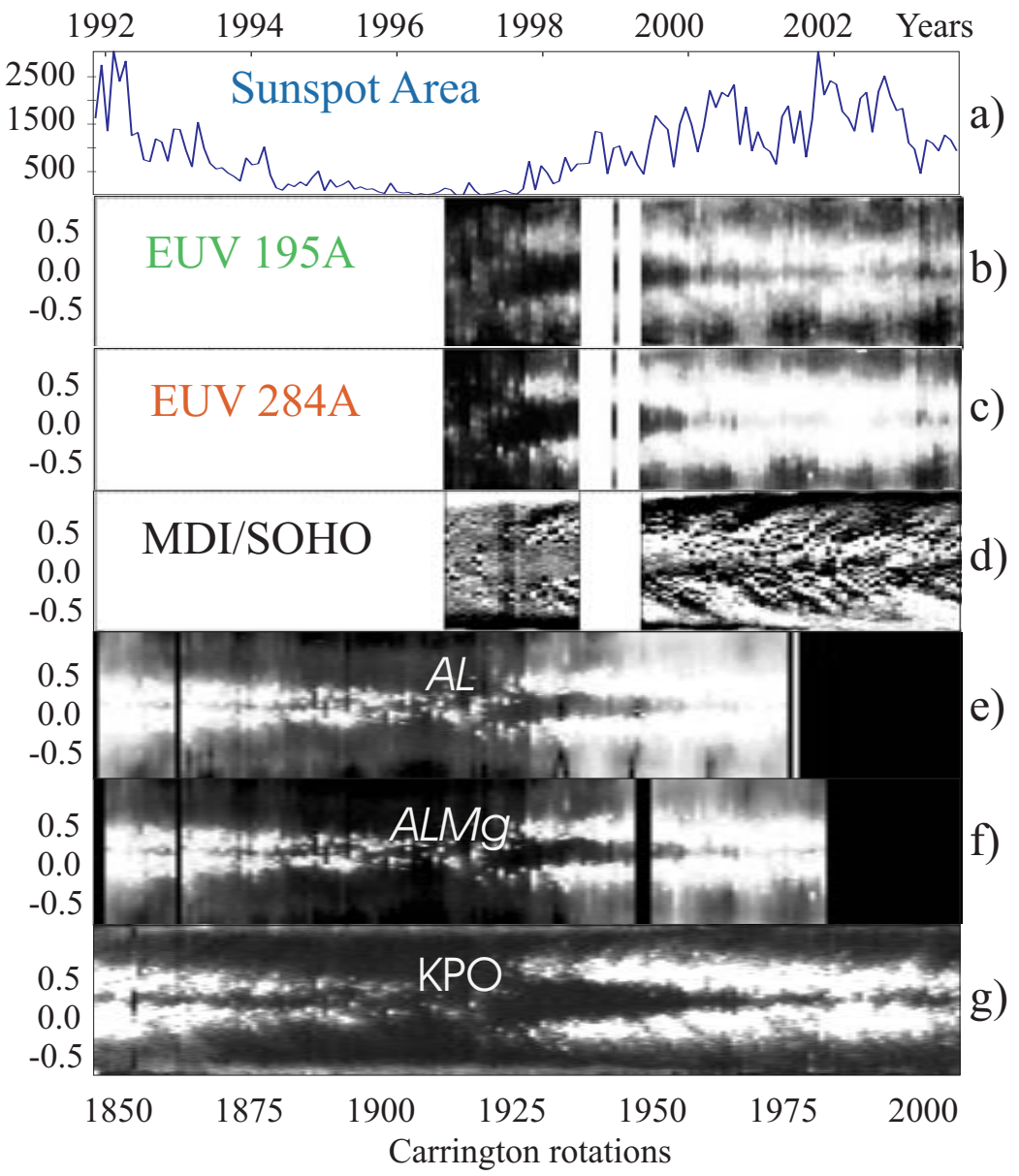

Figure 4. a) Sunspot area as a function of time from 11 November 1991 to 30 August 2003 (Carrington rotations from 1849 to 2006); Axisymmetrical distributions as a function of latitude from $-83^{\circ}$ to $83^{\circ}$ and time for: b)EUV flux in Fe XII and c) Fe XV lines (available only since

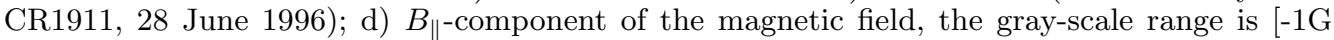
$1 \mathrm{G}]$; e) X-ray flux in the Al filter; f) X-ray flux in the AlMg filter; g) unsigned magnetic flux $\left|B_{\|}\right|$, in the $[020 \mathrm{G}]$ range.

poloidal field is reversed by the alpha-effect rather than by the surface diffusion (Makarov et al. 1987; Belvedere et al. 1991). It has also been found that both low- and highlatitude waves of solar activity may last longer the 11-year sunspot cycle, forming socalled 'extended solar cycle' (e.g. Altrock, 1997).

At present it is unclear whether the processes in the solar corona simply reflect the evolution of the surface or deep fields, or they play an active role in the changes of the magnetic topology due to magnetic reconnection. This question is an important key to understanding the solar cycle.

In our recent papers (Benevolenskaya et al., 2001, 2002) we have identified the coronal activity waves in the extreme ultraviolet data from SOHO/EIT (Delaboudiniere et al., 1995)and soft X-rays from YOHKOH (Tsuneta et al., 1991). It is found, that the bright coronal structures, which migrate to the poles during the rising phase of the solar cycle, are formed by density enhancements in the poleward footpoints of giant loops in EUV emission. These loops can be considered as coronal proxy of a lines of magnetic field lines. These magnetic lines connect the magnetic fields of the following parts of active regions 


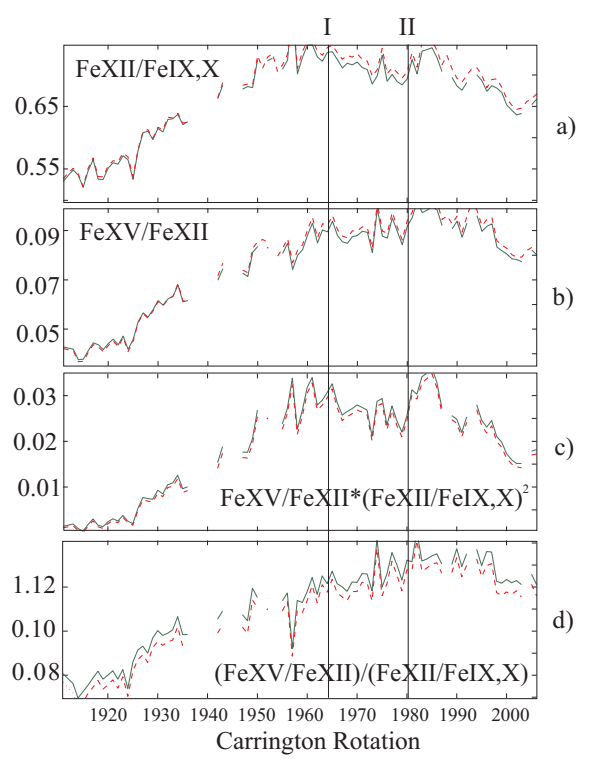

Figure 5. The averaged over latitudes ratios of coronal EUV emissions between lines:

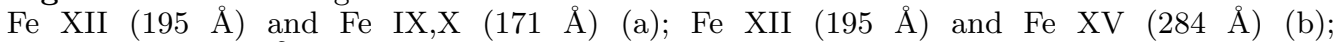
$(F e X I I / F e I X, X)^{2} \cdot(F e X V / F e X I I)(c)$ and $(F e X I I / F e I X, X) /(F e X V / F e X I I)$ (d). Dash line defines the coronal emission averaged over $-70^{\circ}$ to $70^{\circ}$, and solid line does for the the coronal emission averaged over $-75^{\circ}$ to $75^{\circ}$.

with the polar field. In other words, these giant magnetic loops connect the toroidal field of the new solar cycle with the polar poloidal field formed during the previous cycle, providing an important link between these two types of evolving magnetic topology (Figure 6).

Indeed, the EIT time-latitude diagram (for example in line Fe XII, see Figure 4(b)) displays two sets of bright migrating structures in each hemisphere: low-latitude structures that migrate toward the equator following the evolution of $\left|B_{\|}\right|$(low-latitude coronal activity waves or 'butterfly' diagram) and high-latitude structures, or high-latitude waves, that migrate toward the poles parallel to the magnetic neutral lines. The high-latitude coronal structures are located $15-20^{\circ}$ higher in latitude than the neutral line. In the SXT axisymmetrical distributions (Figure $4(e), f)$ ), the low-latitude migrating structures are similar to those in the EIT maps. However, the high-latitude structures look differently, without pronounced brightening in the polar regions, and are more uniform latitudinally, connecting the low-latitude bands with the polar regions.

The EIT images can provide estimates of coronal temperatures from 0.6 to $2 \mathrm{MK}$ (Moses et al., 1997). The EIT estimates of coronal temperature are based on the ratio of the intensities in different Fe lines. We will have to deal with the uncertainties of the temperature diagnostics from the EIT heliograms. Because in active regions the temperature along the line of sight may vary significantly (Altrock et al., 1996) only an effective (averaged) temperature can be estimated. In addition, there is already discussed uncertainty of the temperature diagnostics from the line ratio. Generally, the temperature diagnostics from the fluxes observed in the three channels is an ill-posed mathematical problem. However, the flux ratio provides the information about the some averaged temperature distribution in the corona.

The ratio of the Fe XII (195 $\AA)$ and Fe IX,X (171 $\AA)$ fluxes is predominantly sensitive to coronal structures with the plasma temperature from 1 to $2 \mathrm{MK}$. And the ratio of the 

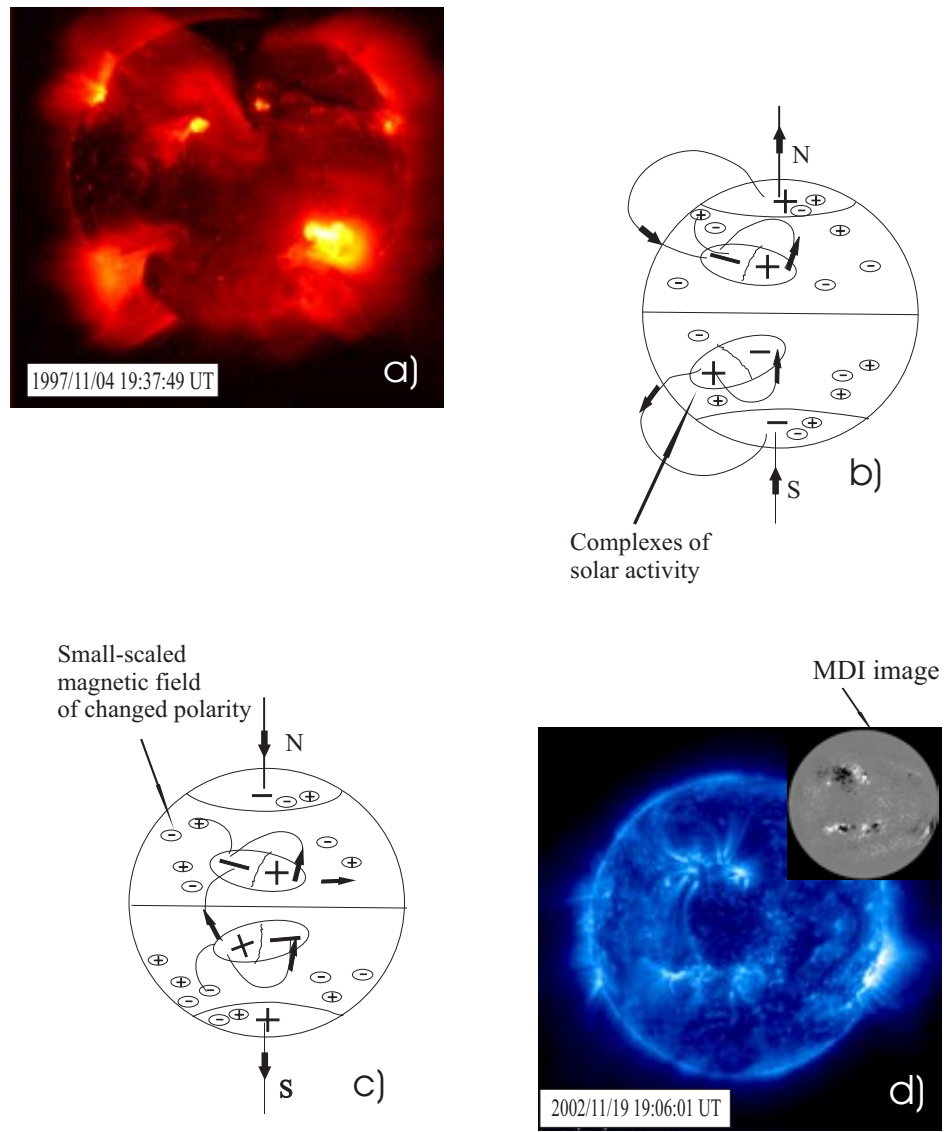

Figure 6. a) X-ray image in AlMg filter, November, 4 1997; b)A schematic picture showing the magnetic connections between the leading and following polarities of active regions and the polar field before the polar magnetic field reversal; c)Picture of the magnetic connection after the polar magnetic field reversals; d) EUV image 171 (Fe IX,X) November, 192002 and MDI image (in upper right corner).

Fe XV $(284 \AA)$ and Fe XII (195 $\AA)$ fluxes is sensitive to coronal structures with more hot plasma heated up to 2.6 MK. Active regions are seen as the hottest structures in these ratio images. The plots of the axi-symmetrical distributions of the ratio for the lines $\mathrm{Fe}$ XII $(195 \AA)$ and Fe IX,X (171 $\AA)$, and for the Fe XV $(284 \AA)$ and Fe XII $(195 \AA)$ are represented in Figure $5(a, b)$, correspondingly. We have applied ratios for the estimation of the low-temperature plasma heated to $1 \mathrm{MK}$ and high-temperature plasma $(\geqslant 2 \mathrm{MK})$ : $(F e X I I / F e I X, X) /(F e X V / F e X I I)$ and $(F e X I I / F e I X, X)^{2} \cdot(F e X V / F e X I I)$ (Figure $5(c, d))$. The high-temperature flux ratio displays the pronounced two-peak structure in cycle 23 , while the low-temperature flux ratio doesn't show it.

\section{Impulsive nature of the solar cycle}

The 'impulses of solar activity', and, in particular, the 'coronal impulses' found in the EIT and SXT data are closely related to long-living complexes of solar activity with arcades of coronal loops. At the beginning of the solar cycle, the magnetic flux appears at the photosphere in the form of bipolar magnetic complexes of activity at the middle latitudes as a sequence of impulses of solar activity. In the corona, we observe the 
magnetic loops connecting regions of opposite polarity inside the bipolar regions, and, in addition, giant loops connecting the following parts of the complexes of activity with the polar regions. Some portion of the erupted magnetic flux energy from the interior contributes to plasma heating in the coronal loops to $2-3 \times 10^{6}$. During the rising phase of the solar cycle, the footpoints of the giant loops in polarward become located closer and closer to the poles following the migrating zonal magnetic neutral lines. When the zonal neutral lines reach the poles then the polar magnetic field changes the polarity. A schematic picture showing the magnetic connections between the leading and following polarities of active regions and the polar field before the polar magnetic field reversal are represented in Figure 6(b). The corresponded soft X-ray image is shown in Figure 6(a). After the polar magnetic field reversals the situation changes. The polar magnetic field has now the same polarity as the following parts of the bi-polar complexes of solar activity (Figure 6(d)). This schematic behavior is represented in Figure 6(c). The connectivity from the following part of complexes of solar activity to the higher latitude zones becomes more random, and is mostly associated with small-scale regions of the opposite polarity, which are persistent over the solar disk together with the large-scale magnetic field. Complexes of solar activity during the declining phase of the solar cycle are located more close to the solar equator; and, transequatorial loops connecting the complexes across the equator predominately appear in the declining phase.

\section{References}

Altrock, R.C., Hick, P., Jackson, B.V., Hoeksema, J.T., Zhao, X.P., Slater, C., Henry, T.W. 1996 Adv. Space Res. 17, 235-238.

Altrock, R.C. 1997 Sol. Phys. 170, 411-423.

Antalova, A., Gnevyshev, M. N. 1985 Astron. Inst. of Czech. Bulletin 36, 61.

Benevolenskaya, E. E. 1991 The sun and Cool Stars: Activity, Magnetism, Dynamos I. Tuominen, D. Moss, and G. Rudiger (eds.) Springer-Verlag, 234.

Benevolenskaya, E. E. 1998, ApJ 509, L49-L52.

Benevolenskaya, E. E., Kosovichev, A. G., Scherrer, P. H. 2001, ApJ 554, L107-L110

Benevolenskaya, E. E., Kosovichev, A. G., Lemen, J. R., Slater G. L., Scherrer, P. H. 2002 ApJ 571, L181-L185.

Babcock, H.M. 1961 ApJ 133, 572-587.

Belvedere, G., Lanzafame, G. \& Proctor, M.R.E. 1991 Nature 350, 481-483.

d'Azambuja, L. 1945 ApJ 101, 260-261.

Delaboudiniere, J.-P., Artzner, G. E., Brunaud, J. et al. 1995 Sol. Phys. 162, 291-312.

Gnevyshev, M. N. 1995 Sol. Phys. 1, 107-120.

Leighton, R.B. 1969 ApJ 156, 1-26.

Leroy, J.L., Trellis, M. 1974 Astron. Astrophys. 35, 283-288.

Makarov, V.I., Ruzmaikin, A.A., \& Starchenko, S.V. 1987 Sol. Phys. 111, 267-277.

Makarov, V. I., Sivaraman, K. R. 1989 Sol. Phys. 123, 367-380.

Moses, D., Clette, F., Delaboudiniere, J.-P. et al. 1989 Sol. Phys. 175, 571-599.

Parker, E.N. 1955 ApJ 122, 293-314.

Rǔsin, V., Rýbansky, M., \& Minarovjech, M. 1998 Synoptic Solar Physics K.S. Balasubramaniam, J.W. Harvey, \& D.M. Rabin (eds) ASP Conf. Ser. 140, 353-361.

Secchi, P.A. 1877 Gauthier-Villars, Paris Vol. 2.

Stenflo,J.O. 1988 ApSS 144, 321-336.

Tsuneta, S.,Acton, L., Bruner, M., Lemen, J., Brown, W., Caravalho, R., Catura, R., Freeland, S., Jurcevich, B., \& Owens, J. Sol.Phys. 136, 37-67.

Waldmeier, M. 1957 Birkäuser, Basel Vol. II.

Wang, Y.-M., Sheeley, N.R., \& Nash, A.G. 1991 ApJ 383, 431-442.

Wilson, P.R., Altrock, R.C., Harvey, K. L., Martin, S.F. Snodgrass, H.B. 1991 Nature 333, 748. 